\title{
Diversity-Fidelity Tradeoff in Transmission of Analog Sources over MIMO Fading Channels
}

\author{
Mahmoud Taherzadeh, Kamyar Moshksar and Amir K. Khandani \\ Coding \& Signal Transmission Laboratory (www.cst.uwaterloo.ca) \\ Dept. of Elec. and Comp. Eng., University of Waterloo \\ Waterloo, ON, Canada, N2L 3G1 \\ Tel: 519-725-7338, Fax: 519-888-4338 \\ e-mail: \{taherzad, kmoshksa, khandani\}@ cst.uwaterloo.ca
}

\begin{abstract}
In this paper, the theoretical limits on the asymptotic performance of joint source-channel coding over MIMO fading channels are investigated. Similar to the concept of Diversity-Multiplexing Tradeoff (DMT) in Digital MIMO systems, a new measure for the asymptotic high-SNR performance of MIMO source-channel codes is introduced, which is called Diversity-Fidelity Tradeoff, and the optimal tradeoff is characterized. Also, the problem of constructing robust MIMO sourcechannel codes, which are not sensitive to the knowledge of the SNR, is investigated and a semi-robust scheme is proposed.
\end{abstract}

\section{INTRODUCTION}

In many applications, such as voice and multimedia transmission in cellular and wireless LAN environments, transmission of analog sources over wireless channels is needed. Results of the research in the past decade shows that using multiple-antenna systems can substantially improve the rate and the reliability of communication in wireless fading environments. However, until now, most of the research has been focused on the transmission of digital data over multiple-input multiple-output (MIMO) channels, not the transmission of analog sources.

For the simpler case of Gaussian channel, without considering the delay limitations, Shannon's source-channel coding separation theorem ensures the optimality of separately designing source and channel codes. However, for the case of a limited delay, even for the Gaussian channel, several articles have shown that joint source-channel codes have a better performance, compared to the separately designed source and channel codes (which are called tandem codes). Thus, designing efficient joint source-channel codes is a key solution for the transmission of analog sources.

Although in recent years there has been a colossal amount of research in MIMO communications, the research on MIMO joint source-channel coding is still in its early stages. In [1] and [2] some digital and hybrid digital-analog techniques are examined for joint source-channel coding over MIMO channels, and some bounds on the asymptotic exponents of the average distortion are presented. However, the asymptotic exponents of the average distortion is not a good measure for the performance evaluation of joint source-channel coding schemes in fading environments. The reason is that the average distortion is dominated by rare cases of very bad channels. In these environments, it is more informative to analyze the probability that distortion is greater than a certain level, instead of averaging very large unusual distortions.

Unlike [1] and [2] which are focused on analyzing the asymptotic exponents of the average distortion, here, we analyze the asymptotic exponents of the probability of having a large distortion. This measure, which we call diversityfidelity tradeoff, can be seen as an analog version of the well-known diversity-multiplexing tradeoff which is proved to be very useful in evaluating various digital space-time coding schemes.

Another concern of this paper is to design robust or semirobust source-channel codes for MIMO fading channels. It is known that digital joint source-channel coding is very sensitive to the mismatch in the estimation of the channel signal-to-noise ratio (SNR). In many cases the exact signalto-noise ratio is not known at the transmitter, and may vary over a large range of values. Two examples of this scenario are transmitting an analog source over a quasistatic fading channel and/or multicasting it to different users (with different channel gains). Therefore, for these kinds of applications, it is very important to have a joint sourcechannel scheme which is robust for a wide range of SNR.

To avoid the saturation effect of digital coding, hybrid digital-analog codes are proposed in the past. Although these codes can provide asymptotic gains (for high SNR) over the simple repetition code, they suffer from a threshold effect. Indeed, when the SNR becomes less than a certain threshold, the performance of these systems degrades severely. Therefore, the parameters of these methods should be chosen according to the operating SNR, hence, these methods are still very sensitive to the errors in the estimation of SNR. Also, although the performance of the system is not saturated for the high SNR (unlike digital codes), the scaling of the end-to-end distortion is far from the theoretical bounds.

In [3], for the case of Gaussian channel, a joint sourcechannel coding scheme is introduced which has a robust performance over the entire range of SNR. Unlike the previous methods, the method asymptotically achieves the optimal scaling of the signal-to-distortion-ratio (SDR) without being affected by the threshold effect. In this paper we generalize the idea of this scheme for the case of MIMO channels to construct semi-robust joint source-channel codes for point- 
to-point MIMO systems.

\section{SySTEM MODEL}

We consider a communication system where an analog source of Gaussian independent samples with variance $\sigma_{s}^{2}$ is to be transmitted over a $\left(N_{t}, N_{r}\right)$ block fading MIMO channel where $N_{t}$ and $N_{r}$ are the number of transmit and receive antennas respectively. Every $m$ samples of the source stacked in a vector $\mathbf{x}_{s}$ are transmitted over $n$ channel uses. The channel matrix $\mathbf{H}$ is assumed fixed during this period and changes independently for the next $n$ channel uses. We call the ratio $\eta=\frac{n}{m}$ the expansion/contraction factor of the system. In a general setting, the communication strategy consists of source/channel coding and source/channel decoding. As a result of source channel coding, $\mathbf{x}_{s}$ is mapped into a $N_{t} \times n$ space-time matrix $\mathbf{X}$ which in turn is received at the receiver side as an $N_{r} \times n$ matrix $\mathbf{Y}$ where

$$
\mathbf{Y}=\sqrt{\frac{\mathrm{SNR}}{N_{t}}} \mathbf{H X}+\mathbf{W}
$$

in which SNR is the average signal to noise ratio at each receive antenna, and $\mathbf{W}$ is the additive noise matrix at the receiver whose entries are taken to be $\mathcal{C N}(0,1)$. At the receiver side, the source/channel decoder yields an estimation of $\mathbf{x}_{s}$ out of $\mathbf{Y}$ as $\widehat{\mathbf{x}_{s}}$. For a specific channel realization $\mathbf{H}$, the distortion measure is

$$
D(H)=\mathrm{E}_{\mathbf{x}_{s}}\left\{\left\|\mathbf{x}_{s}-\widehat{\mathbf{x}_{s}}\right\|^{2} \mid \mathbf{H}\right\} .
$$

For any specific strategy, we define the $f$-fidelity event as $\mathcal{A}(f)=\left\{\mathbf{H}: D(\mathbf{H})>\mathrm{SNR}^{-f}\right\}$ and we call $f$ the fidelity exponent. For specific values of $\eta, N_{t}$ and $N_{r}$, we define:

$$
d(f)=\lim _{\mathrm{SNR} \rightarrow \infty}-\frac{\log \operatorname{Pr}\{\mathcal{A}(f)\}}{\log \mathrm{SNR}}
$$

We call $d(f)$ the diversity, and denote its maximum (over all possible source-channel coding schemes) as $d^{*}(f)$.

\section{DIVERSITY-FIDELITY TRADEOFF}

In this section, we characterize the tradeoff between the fidelity exponent $f$ and the optimum diversity $d^{*}(f)$.

\section{A. Upper bound on $d(f)$}

We recall from [4] that if we denote the eigenvalues of $\mathbf{H H}^{\mathrm{H}}$ by $\lambda_{i}$, setting $\alpha_{i}=-\frac{\log \lambda_{i}}{\log \mathrm{SNR}}$, we have ${ }^{1}$ :

$$
p(\vec{\alpha}) \doteq \mathrm{SNR}^{-\sum\left(2 i-1+\left|N_{t}-N_{r}\right|\right) \alpha_{i}} .
$$

On the other hand, in a system of tandem coder, i.e., separate source coder and channel coder, we have:

$$
\operatorname{Pr}\{\operatorname{error} \mid \mathbf{H}\} \leq \mathrm{SNR}^{-n\left[\sum\left(1-\alpha_{i}\right)^{+}-r\right]}
$$

where $R=r \log \mathrm{SNR}$ is the transmission rate over the channel, i.e., $R=\frac{\log M}{n}$, and $M$ is the number of quantized points in the output of the source coder.

\footnotetext{
${ }^{1}$ In this paper, we use $a \doteq b$ to denote that $a$ and $b$ are asymptotically equivalent.
}

To get an upper bound on $d(f)$, we consider the case of delay unlimited where $m, n \rightarrow \infty$ and $\frac{n}{m}=\eta$ is a constant. Also, we assume that the transmitter has perfect knowledge of the channel matrix $H$, and therefore, one may talk about the capacity of this MIMO channel which is given by [4]:

$$
\begin{gathered}
R=\sup _{\Sigma: \operatorname{tr}(\Sigma) \leq m} \log \operatorname{det}\left(\mathbf{I}+\frac{\mathrm{SNR}}{m} \mathbf{H} \Sigma \mathbf{H}^{\mathrm{H}}\right) \\
\leq \log \operatorname{det}\left(\mathbf{I}+\mathrm{SNRHH}^{\mathrm{H}}\right) .
\end{gathered}
$$

We know that the source rate is $R_{s}=\eta R$. On the other hand, the Distortion-Rate function of the source is $\mathcal{D}\left(R_{s} ; \mathbf{H}\right)=e^{-2 R_{s}}$. Therefore:

$$
\mathcal{D}\left(R_{s} ; \mathbf{H}\right) \geq \frac{1}{\operatorname{det}\left(\mathbf{I}+\mathrm{SNRHH}^{\mathrm{H}}\right)^{2 \eta}} .
$$

Let us denote the $f$-fidelity event as $\mathcal{A}_{\infty}(f)$ here. Thus, we obtain:

$$
\begin{aligned}
& \operatorname{Pr}\left\{\mathcal{A}_{\infty}(f)\right\}=\operatorname{Pr}\left\{\mathcal{D}\left(R_{s} ; H\right)>f\right\} \geq \\
& \operatorname{Pr}\left\{\frac{1}{\operatorname{det}\left(\mathbf{I}+\mathrm{SNRHH}^{\mathrm{H}}\right)^{2 \eta}}>\mathrm{SNR}^{-f}\right\}
\end{aligned}
$$

which can be written as:

$$
\begin{aligned}
\operatorname{Pr}\left\{\mathcal{A}_{\infty}(f)\right\} & \geq \operatorname{Pr}\left\{\prod_{i=1}^{i=n}\left(1+\operatorname{SNR} \lambda_{i}\right)<\operatorname{SNR}^{\frac{f}{2 \eta}}\right\} \\
& \doteq \operatorname{Pr}\left\{\sum_{i}\left(1-\alpha_{i}\right)^{+}<\frac{f}{2 \eta}\right\}
\end{aligned}
$$

As a result, based on (3) and (10), we get:

$$
\operatorname{Pr}\left\{\mathcal{A}_{\infty}(f)\right\} \geq \int_{\vec{\alpha} \in \Delta} \mathrm{SNR}^{-\sum\left(2 i-1+\left|N_{t}-N_{r}\right|\right) \alpha_{i}} d \vec{\alpha}
$$

where $\Delta=\left\{\vec{\alpha}: \sum_{i}\left(1-\alpha_{i}\right)^{+}<\frac{f}{2 \eta}\right\}$. Based on [4], we have:

$$
\int_{\vec{\alpha} \in \Delta} \mathrm{SNR}^{-\sum\left(2 i-1+\left|N_{t}-N_{r}\right|\right) \alpha_{i}} d \vec{\alpha}=\mathrm{SNR}^{-d_{u b}(f)}
$$

where $d_{u b}(f)=\min \vec{\alpha} \in \Delta \sum\left(2 i-1+\left|N_{t}-N_{r}\right|\right) \alpha_{i}$. According to the results in [4], for integer values of $\frac{f}{2 \eta}$, this can be calculated as:

$$
d_{u b}(f)=\left(N_{t}-\frac{f}{2 \eta}\right)\left(N_{r}-\frac{f}{2 \eta}\right) .
$$

Clearly, if we let $\operatorname{Pr}\left\{\mathcal{A}_{\infty}(f)\right\} \doteq \mathrm{SNR}^{-d_{\infty}(f)}$, we have $d_{\infty}(f) \leq d_{u b}(f)$. Consequently, $d_{u b}(f)$ is an upper bound on $d(f)$. 


\section{B. Achievability of the optimal tradeoff}

We show that for any given bandwidth expansion $\eta=\frac{n}{m}$, the upper bound for $d^{*}(f)$ is tight and it can be achieved by a family of delay-limited digital space-time codes $\left\{\mathcal{C}_{k}\right\}$. We map $m N_{t}^{2}$ samples of the source to an $n N_{t}^{2}$-dimensional vector $\mathbf{s}=\left[s_{1} \ldots s_{n N_{t}^{2}}\right]^{\mathrm{T}}$, and construct the MIMO codeword by using $n$ consecutive $N_{t} \times N_{t}$ space-time matrices $\left\{\mathcal{C}_{k}\right\}$, chosen from a DMT-achieving family of digital codes (for example, space-time code with the non-vanishing determinant property, e.g. Perfect Codes [5] [6] [7]).

For the modulating signal $\mathbf{x}_{s}=\left(x_{1}, \ldots, x_{m N_{t}^{2}}\right)$, consider $x_{i}+\frac{1}{2}=\left(\overline{0 . b_{i, 1} b_{i, 2} b_{i, 3} \cdots}\right)_{2}$. Let $b_{i+(j-1) m N_{t}^{2}}^{\prime}=b_{i, j}$. For the code $\mathcal{C}_{k}$, we construct $s_{1}, s_{2}, \ldots, s_{N}$ (where $N=n N_{t}^{2}$ ) as

$$
\begin{gathered}
s_{1}=\left(\overline{0 . b_{1}^{\prime} b_{N+1}^{\prime} b_{2 N+1}^{\prime} \ldots b_{(k-1) N+1}^{\prime}}\right)_{2}, \\
s_{2}=\left(\overline{0 . b_{2}^{\prime} b_{N+2}^{\prime} b_{2 N+2}^{\prime} \ldots b_{(k-1) N+2}^{\prime}}\right)_{2}, \\
\ldots \\
s_{N}=\left(\overline{0 . b_{N}^{\prime} b_{2 N}^{\prime} b_{3 N}^{\prime} \ldots b_{k N}^{\prime}}\right)_{2},
\end{gathered}
$$

Theorem 1 For the proposed family of codes, if we use $\mathcal{C}_{k}$ for $2^{-(k-1) N_{t}}>\mathrm{SNR}^{-\frac{f}{2 \eta}} \geq 2^{-k N_{t}}$,

$$
d(f)=\left(N_{t}-\frac{f}{2 \eta}\right)\left(N_{r}-\frac{f}{2 \eta}\right)
$$

for integer values of $\frac{f}{2 \eta}$.

Proof: Because the family of codes $\mathcal{C}_{k}$ are obtained from a lattice with non-vanishing determinant property, using ML decoding, they achieve the optimal diversity-multiplexing tradeoff. Thus, for this family of codes,

$$
P_{\text {error }}(r) \doteq \mathrm{SNR}^{-\left(N_{t}-r\right)\left(N_{r}-r\right)}
$$

for integer values of $r$, the normalized rate $\left(r=\frac{R}{\log \mathrm{SNR}}\right)$. In this scheme, code $\mathcal{C}_{k}$ has rate $R_{k}=k N_{t}$ and is used for $2^{-(k-1) N_{t}}>\mathrm{SNR}^{-\frac{f}{2 \eta}} \geq 2^{-k N_{t}}$. Therefore, $r \doteq \frac{f}{2 \eta}$, hence,

$$
P_{\text {error }}(r) \doteq \mathrm{SNR}^{-\left(N_{t}-\frac{f}{2 \eta}\right)\left(N_{r}-\frac{f}{2 \eta}\right)} .
$$

When there is no error in decoding $\mathcal{C}_{k}$, the $k N=$ $k n N_{t}^{2}$ bits $b_{1}^{\prime}, \ldots, b_{k N}^{\prime}$ are decoded correctly, hence the first $\left\lfloor k \frac{n}{m} N_{t}^{2}\right\rfloor=\lfloor k N \eta\rfloor$ bits of $x_{i}$ can be reconstructed without error (for $1 \leq i \leq m$ ), hence $D \doteq 2^{-2 k N \eta} \doteq \mathrm{SNR}^{-f}$. Therefore,

$$
\begin{gathered}
\operatorname{Pr}\left\{D>\mathrm{SNR}^{-f}\right\} \leq P_{\text {error }}(r) \\
\quad \doteq \mathrm{SNR}^{-\left(N_{t}-\frac{f}{2 \eta}\right)\left(N_{r}-\frac{f}{2 \eta}\right)} \\
\Longrightarrow d(f) \geq\left(N_{t}-\frac{f}{2 \eta}\right)\left(N_{r}-\frac{f}{2 \eta}\right) .
\end{gathered}
$$

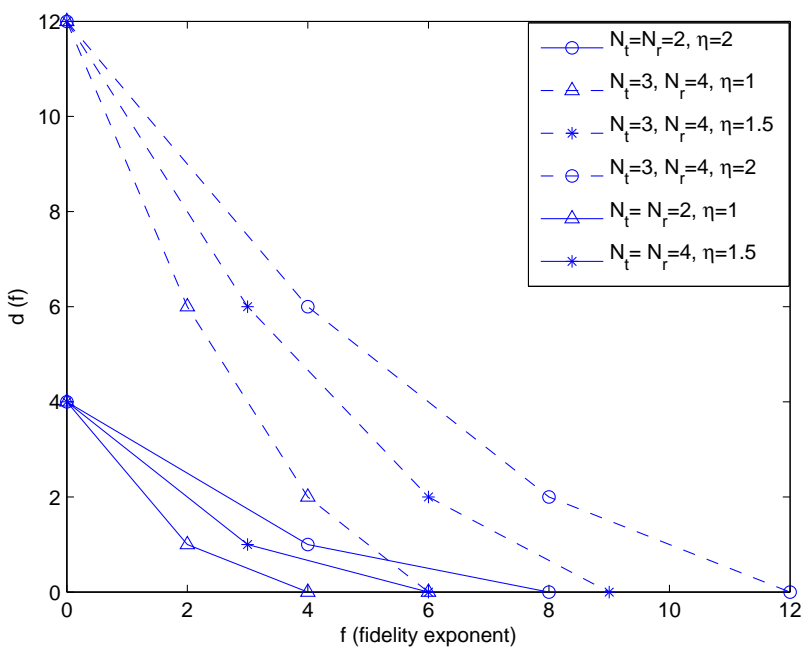

Fig. 1. Diversity-Fidelity Tradeoff for different numbers of antennas and different bandwidth expansion factors

\section{ROBUST SPACE-TIME SOURCE-CHANNEL CODING}

In the scheme presented in the previous section, for the achievability of the optimal tradeoff, different codes should be used for different values of distortion $D$ and the channel SNR.

In this section, we consider the problem of designing robust or semi-robust codes which can work for the entire range of SNR.

The simplest non-trivial case is the case two transmit antennas and one receive antenna. In this special case, using Alamouti scheme at the transmitter and simple Alamouti decoding (which is equivalent to zero forcing) can easily separate the encoded symbols. Indeed, for the case of $\eta=1$ and a Gaussian source, the Alamouti scheme is optimum, not only in SDR scaling, but in achieving the exact optimal performance.

Theorem 2 Consider a $2 \times 1$ MIMO system and the bandwidth expansion $\eta=1$ a Gaussian i.i.d source $x_{i}$. If we send the Alamouti scheme to send consecutive source samples $x_{1}, x_{2}$ over two consecutive channel uses, and use the Alamouti detection (zero forcing) at the transmitter, for any noise variance $\sigma_{z}$, the resulting distortion is the minimum possible distortion.

Proof: Let $\mathbf{H}=\left[\begin{array}{ll}h_{1} & h_{2}\end{array}\right]$ be the channel matrix. When we do not have the channel state information (CSI) at the transmitter, the best power allocation scheme at the transmitter is to assign equal power to the transmit antennas. In this case, for any realization of the channel, the capacity of the $2 \times 1$ channel is equal to [8]

$$
\begin{gathered}
C(\mathbf{H})=\frac{1}{2} \log \operatorname{det}\left(\mathbf{I}_{2}+\frac{S N R}{2} \mathbf{H I}_{2} \mathbf{H}^{\mathrm{H}}\right) \\
=\log \left(1+\frac{S N R}{2}\left(\left|h_{1}\right|^{2}+\left|h_{2}\right|^{2}\right)\right) .
\end{gathered}
$$


If the source has i.i.d. Gaussian distribution, according to the rate-distortion function of the Gaussian source,

$$
D_{\text {min }}=\frac{2}{S N R\left(\left|h_{1}\right|^{2}+\left|h_{2}\right|^{2}\right)} .
$$

On the other hand, if we send the analog source samples directly by using the Alamouti scheme,

$$
\begin{aligned}
\mathbf{Y} & =\sqrt{\frac{S N R}{2}} \mathbf{H X}+\mathbf{W}=\left[h_{1} h_{2}\right]\left[\begin{array}{cc}
x_{1} & x_{2} \\
-x_{2}^{*} & x_{1}^{*}
\end{array}\right]+\left[\begin{array}{ll}
w_{1} & w_{2}
\end{array}\right] \\
& \Longrightarrow\left[y_{1} y_{2}^{*}\right]=\sqrt{\frac{S N R}{2}}\left[x_{1} x_{2}\right]\left[\begin{array}{cc}
h_{1} & h_{2} \\
h_{2}^{*} & -h_{1}^{*}
\end{array}\right]+\left[\begin{array}{ll}
w_{1} & w_{2}^{*}
\end{array}\right]
\end{aligned}
$$

By using the Alamouti decoding (which is equivalent to zero forcing), the received signal is equal to:

$$
\begin{gathered}
{\left[\widetilde{x}_{1} \widetilde{x}_{2}\right]=\left[x_{1} x_{2}\right]+\sqrt{\frac{2}{S N R}}\left[w_{1} w_{2}^{*}\right]\left[\begin{array}{cc}
h_{1} & h_{2} \\
h_{2}^{*} & -h_{1}^{*}
\end{array}\right]^{-1}} \\
\Longrightarrow D_{\text {Alamouti }}=\frac{2}{S N R\left(\left|h_{1}\right|^{2}+\left|h_{2}\right|^{2}\right)}=D_{\text {min }}
\end{gathered}
$$

For other cases of $\eta \neq 1$ using the bandwidth expansion mappings in [3] can achieve the optimal tradeoff. However, for other configurations of MIMO system, no optimum symbol-separating scheme such as the Alamouti scheme (which can work with analog symbols) is known.

Here we propose a semi-robust coding scheme that achieves a diversity order, larger than 1 (better than the trivial linear SISO code or MMSE-decoded V-BLAST). We map $m k N_{t}$ samples of the source to an $n k N_{t}$-dimensional vector $\mathbf{s}=\left[s_{1} \ldots s_{n k N_{t}}\right]^{\mathrm{T}}$ (for an inter $k, 1 \leq k \leq N_{t}$ ), using a robust point-to-point joint source-channel code, and construct the MIMO codeword by setting $\mathbf{C}=\left(\mathbf{L}_{N_{t}^{2} \times k N_{t}} \otimes \mathbf{I}_{n}\right) \mathbf{s}$ (where $\mathbf{L}_{N_{t}^{2} \times k N_{t}}$ is the generator of a lattice space-time code with non-vanishing determinant property) and mapping it to the entries of $n$ consecutive $N_{t} \times N_{t}$ space-time matrices. To construct the point-to-point joint source-channel code for the bandwidth expansion of $\eta=\frac{n N_{t}^{2}}{m k N_{t}}$, we generalize the codes presented in the [9] and [3]:

For the modulating signal $\mathbf{x}_{s}=\left(x_{1}, \ldots, x_{m k N_{t}}\right)$, consider $x_{i}+\frac{1}{2}=\left(\overline{0 . b_{i, 1} b_{i, 2} b_{i, 3} \cdots}\right)_{2}$. Let $b_{i+(j-1) m k N_{t}}^{\prime}=b_{i, j}$ (for $\left.1 \leq i<m k N_{t}\right)$. We construct $s_{1}, s_{2}, \ldots, s_{N}$ (where $N=$ $\left.n k N_{t}\right)$ as

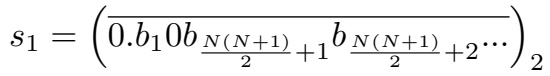

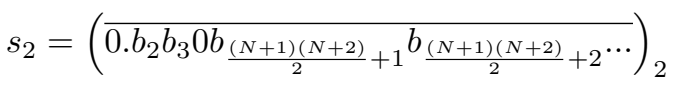

$$
\begin{aligned}
& \text {... }
\end{aligned}
$$

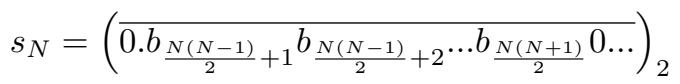

The resulting code $\mathcal{C}$ can be seen as successively refining space-time code with infinite layers of refinement.

At the receiver, we use simple linear decoding to separate $s_{1}, s_{2}, \ldots, s_{N}$ and decode them separately. To analyze the Diversity-fidelity tradeoff of this single mapping, we first lower bound the probability that the received effective noise (after zero forcing) becomes large. The received signal (over $n N_{t}$ channel uses and $N_{r}$ receive antennas) can be represented as a $n N_{r} N_{t} \times 1$ vector $\mathbf{y}=$ $\left(\mathbf{H} \otimes \mathbf{I}_{n N_{t}}\right)\left(\mathbf{L}_{N_{t}^{2} \times k N_{t}} \otimes \mathbf{I}_{n}\right) \mathbf{s}+\mathbf{w}_{n N_{t} N_{r}}$ where $\mathbf{w}_{n N_{t} N_{r}}$ is the vector representation for the Gaussian noise in $n N_{t}$ channel uses. At the receiver, we can multiply the pseudoinverse of the matrix $\left(\mathbf{H} \otimes \mathbf{I}_{n N_{t}}\right)\left(\mathbf{L}_{N_{t}^{2} \times k N_{t}} \otimes \mathbf{I}_{n}\right)$ to find an estimate of $s_{1}, s_{2}, \ldots, s_{N}$.

Lemma 1 Consider $\mathbf{L}_{N_{t}^{2} \times k N_{t}}$ as generator of an $N_{t} \times N_{t}$ lattice code (with dimension $k N_{t}$ ) with non-vanishing determinant property. The probability that the amplitude of effective received noise is larger $\varepsilon$ is upper bounded by

$$
\operatorname{Pr}\left\{\left|\mathbf{w}^{\prime}\right|^{2} \geq \frac{1}{\varepsilon}\right\}<c \varepsilon^{\left(N_{t}-k+1\right)\left(N_{r}-k+1\right)}
$$

for some constant $c$.

Sketch of the proof: After multiplying by the pseudoinverse of the equivalent channel matrix,

$$
\mathbf{w}^{\prime}=\left(\left(\mathbf{H} \otimes \mathbf{I}_{n N_{t}}\right)\left(\mathbf{L}_{N_{t}^{2} \times k N_{t}} \otimes \mathbf{I}_{n}\right)\right)^{+} \mathbf{w} .
$$

The probability that $\left|\mathbf{w}^{\prime}\right|^{2} \geq \frac{1}{\varepsilon}$ can be bounded by the summation of the probability the Frobenius norm of $\left(\mathbf{H} \otimes \mathbf{I}_{N_{t}}\right) \mathbf{L}_{N_{t}^{2} \times k N_{t}}$ is greater than $\frac{c_{1}}{\varepsilon^{2}}$ for a constant $c_{1}$ and the probability that $|\mathbf{w}|^{2}$ is larger than $\frac{1}{c_{1}}$. we can choose $c_{1}$ such that the first term of the summation becomes dominant. Now, by using the non-vanishing-determinant property of $\mathbf{L}$ and the fact that the entries of $\mathbf{H}$ have independent Gaussian distributions, we can bound the second term by $c_{2} \varepsilon^{\left(N_{t}-k+1\right)\left(N_{r}-k+1\right)}$, for some constant $c_{2}$.

Theorem 3 If we use the proposed scheme, consisting of the SISO source-channel code (27) and a $N_{t} \times N_{t}$ space-time code of rate $k$ (constructed by a lattice generator $\mathbf{L}_{N_{t}^{2} \times k N_{t}}$ for an integer $k, 1 \leq k \leq \min \left\{N_{t}, N_{r}\right\}$ ) with non-vanishing determinant property, and use zero forcing at the receiver, the diversity-fidelity tradeoff of the scheme is lower bounded by

$$
\begin{aligned}
d(f) \geq\left(N_{t}-k+1\right)\left(N_{r}-k+1\right)- & \\
& \frac{\left(N_{t}-k+1\right)\left(N_{r}-k+1\right)}{k} \frac{f}{2 \eta} .
\end{aligned}
$$

Sketch of the proof: Because the bits of the source symbols are almost uniformly distributed among $s_{1}, s_{2}, \ldots, s_{N}$, to have $D>S N R^{-f}$, at the decoder, at least one of the modulated symbols $s_{i}$ must see a noise larger than $S N R^{-\frac{f}{2 \eta}}$ 
and for that, we should have $\left|\mathbf{w}^{\prime}\right|^{2} \geq S N R \times S N R^{-\frac{f}{2 \eta}}=$ $S N R^{1-\frac{f}{2 \eta}}$. Now, using the result of lemma 1 and the definition of $\mathrm{d}(\mathrm{f})$,

$$
\begin{aligned}
d(f) \geq\left(N_{t}-k+1\right)\left(N_{r}-k+1\right)- & \\
& \frac{\left(N_{t}-k+1\right)\left(N_{r}-k+1\right)}{k} \frac{f}{2 \eta} .
\end{aligned}
$$

\section{CONCLUSIONS}

Similar to the diversity-multiplexing tradeoff which is well known for the high-SNR asymptotic performance of space-time codes, diversity-fidelity tradeoff can be used as a benchmark for evaluating various MIMO source-channel codes. This tradeoff can be achieved by a simple modification of the well-known DMT-achieving codes. However, though the optimum tradeoff can be achieved for different numbers of antennas and arbitrary bandwidth expansion factors, this well-known approach is not robust (design of the codes depend on the SNR of the channel). In this paper, a semi-robust MIMO source-channel scheme is proposed that achieves nontrivial diversity and fidelity exponents for different numbers of antennas. However, the semi-robust scheme that is presented in this paper only achieves the optimum tradeoff for $N_{r}=1$ or $N_{t}=1$ and in the general case of $N_{r}>1$ and $N_{t}>1$, its tradeoff curve is suboptimum. Therefore, the problem of achieving the optimum diversity-fidelity tradeoff by practical and robust codes remains open.

\section{REFERENCES}

[1] D. Gunduz and E. Erkip, "Distortion exponents of mimo fading channels," in IEEE Information Theory Workshop, p. 694698, July 2006.

[2] G. Caire and K. Narayanan, "On the distortion SNR exponent of hybrid digital analog space time coding," IEEE Trans. Info. Theory, vol. 53, pp. 2867 - 2878, August 2007.

[3] M. Taherzadeh and A. K. Khandani, "Robust joint source-channel coding for delay-limited applications," in ISIT 2007, (NiceFrance), June 2007.

[4] L. Zheng and D. Tse, "Diversity and multiplexing: a fundamental tradeoff in multiple-antenna channels," IEEE Trans. Info. Theory, pp. 10731096, May 2003.

[5] P. Elia, K. R. Kumar, S. A. Pawar, and P. V. K. H.-F. Lu, "Explicit spacetime codes achieving the diversitymultiplexing gain tradeoff," IEEE Trans. Info. Theory, vol. 52, pp. 3869-3884, Sep. 2006.

[6] F. Oggier, G. Rekaya, J.-C. Belfiore, and E. Viterbo, "Perfect spacetime block codes," IEEE Trans. Info. Theory, vol. 52, pp. 3885-3902, Sep. 2006.

[7] L. Hsiao-Feng and P. V. Kumar, "A unified construction of space-time codes with optimal rate-diversity tradeoff," IEEE Trans. Info. Theory, vol. 51, pp. 1709-1730, May 2005.

[8] I. E. Telatar, "Capacity of multi-antenna gaussian channels," Europ. Trans. Telecommun., pp. 585-595, Nov. 1999.

[9] M. Taherzadeh and A. K. Khandani, "Analog coding for delay-limited applications," in Proceedings of 41 st Conference on Information Sciences and Systems, March 2007. 